Acquired alexithymia following damage to the anterior insula

J. Hogeveen ${ }^{1,2, *}$, G. Bird ${ }^{3,4}$, A. Chau ${ }^{1}$, F. Krueger ${ }^{5,6}$, \& J. Grafman ${ }^{1,2,7^{\wedge}}$

1. Cognitive Neuroscience Laboratory, Rehabilitation Institute of Chicago, Chicago, IL, USA,

2. Department of Physical Medicine and Rehabilitation, Feinberg School of Medicine, Northwestern University, Chicago, IL, USA,

3. MRC Social, Genetic, and Developmental Psychology Centre, King's College London, London, United Kingdom,

4. Institute of Cognitive Neuroscience, University College London, London, United Kingdom,

5. Molecular Neuroscience Department, George Mason University, Fairfax, VA, USA,

6. Department of Psychology, George Mason University, Fairfax, VA, USA,

7. Department of Neurology, Feinberg School of Medicine, Northwestern University.

${ }^{*}$ Correspondence to: Dr. Jeremy Hogeveen, Cognitive Neuroscience Laboratory, Rehabilitation Institute of Chicago, 345 E Superior St., Chicago, IL, 60611. Email: jeremy.hogeveen@northwestern.edu.

^Note: Questions regarding the Vietnam Head Injury Study can be directed to Dr. Jordan Grafman, E-mail: jgrafman@northwestern.edu. 


\begin{abstract}
Alexithymia is a subclinical condition characterized by impaired awareness of one's emotional states, which has profound effects on mental health and social interaction. Despite the clinical significance of this condition, the neurocognitive impairment(s) that lead to alexithymia remain unclear. Recent theoretical models suggest that impaired anterior insula (Al) functioning might be involved in alexithymia, but conclusive evidence for this hypothesis is lacking. We measured alexithymia levels in a large sample of brain-injured patients $(N=129)$ and nonbrain-injured control participants $(N=33)$, to determine whether alexithymia can be acquired after pronounced damage to the Al. Alexithymia levels were first analyzed as a function of group, with patients separated into four groups based on $\mathrm{Al}$ damage: patients with $>15 \%$ damage to $\mathrm{Al}$, patients with $<15 \%$ damage to $\mathrm{Al}$, patients with no damage to $\mathrm{Al}$, and healthy controls. An ANOVA revealed that alexithymia levels varied across groups $(p=0.009)$, with $>15 \% \mathrm{Al}$ damage causing higher alexithymia relative to all other groups (all $p<0.01)$. Next, a multiple linear regression model was fit with the degree of damage to $\mathrm{Al}$, the degree of damage to a related region (the anterior cingulate cortex, ACC), and the degree of damage to the whole brain as predictor variables, and alexithymia as the dependent variable. Critically, increased Al damage predicted increased alexithymia after controlling for the other two regressors (ACC damage; total lesion volume). Collectively, our results suggest that pronounced Al damage causes increased levels of alexithymia, providing critical evidence that this region supports emotional awareness.
\end{abstract}


Keywords: Alexithymia, Anterior Insula, Anterior Cingulate Cortex, Human Lesion Study, Interoception. 


\section{Acquired alexithymia following damage to the anterior insula}

Alexithymia is a subclinical condition characterized by impaired awareness of one's emotional states (Aleman, 2005; Bagby et al., 1994; Nemiah et al., 1976; Taylor et al., 1991). The emotional symptoms of a variety of disorders have been linked to co-occurring alexithymia, such as autism (Bird et al., 2010), eating disorders (Brewer et al., 2015a), and schizophrenia (Fogley et al., 2014), suggesting that intact awareness of one's own emotions might be critical to mental health. The potential translational value of this work has led to an increasing interest in alexithymia both in the public (Robson, 2015; Serani, 2014) and scientific (Bird and Cook, 2013; Lane et al., 2015) literatures, yet the neurocognitive impairments that lead to alexithymia remain unclear.

Recent theoretical work suggests that alexithymia might be the result of impaired "interoception," referring to one's sensitivity to the internal state of one's own body (Gu et al., 2013; Quattrocki and Friston, 2014; Seth, 2013). According to these theories, interoception involves the continual generation of predictive models of the internal state of the body, which are tested against incoming sensory evidence. Discrepancies between predictions and evidence are referred to as "prediction errors," which are hierarchically organized, with low-level errors representing basic somatosensory signals, which are used to update increasingly higher-level models. At the highest level of the interoceptive prediction hierarchy, the anterior insula $(\mathrm{Al})$ and anterior cingulate cortex $(\mathrm{ACC})$ are believed to be 
involved in representing our conscious emotional experience. In particular, Al has a clearly ascribed functional role in this framework: it generates awareness of one's emotions by integrating prediction errors across lower levels of the hierarchy (Gu et al., 2013; Seth, 2013).

Several lines of evidence suggest that $\mathrm{Al}$ abnormalities might be a neurophysiological factor contributing to alexithymia. Specifically, alexithymia is associated with reduced $\mathrm{Al}$ activation when rating the emotional valence of stimuli from the International Affective Pictures System (Silani et al., 2008), and when observing emotional facial expressions (Kano et al., 2003; Reker et al., 2010) and others in pain (Bird et al., 2010; FeldmanHall et al., 2013). In addition to these functional differences, recent structural neuroimaging studies (i.e. voxelbased morphometry) found reduced grey matter volumes of the Al in alexithymic individuals (Borsci et al., 2009; Ihme et al., 2013), and reduced coherence of anterior-insula based anatomical networks (Bernhardt et al., 2014). In accordance with the presence of $\mathrm{Al}$ abnormalities in alexithymia, and its role in interoception, a recent behavioral study found a negative association between interoceptive awareness and the emotional symptoms of alexithymia (Herbert et al., 2011). Collectively, such findings have led to the hypothesis that abnormal recruitment of the Al signals impaired integration of interoceptive prediction errors, which might lead to deficient emotional awareness in alexithymia (Brewer et al., 2015b; Quattrocki and Friston, 2014). 
Research on the neural basis of alexithymia has traditionally focused on individuals whose alexithymic personality features arose over the course of development, which cannot determine whether any of these neurocognitive features reflect an underlying cause of the condition. In contrast, human lesion studies hold the potential to establish a causal link between particular brain regions and alexithymia. There is some evidence for acquired alexithymia following brain injury (Henry et al., 2006; Williams and Wood, 2013, 2010). However, no studies have analyzed alexithymia as a function of lesion location, making it impossible to link alexithymia to Al damage specifically. Accordingly, we measured alexithymia in a large sample of patients with well-localized penetrating traumatic brain injuries $(N=129$ total) and closely matched control participants $(N=33)$ as part of the Vietnam Head Injury Study (VHIS; Raymont et al., 2011). We predicted that patients with pronounced Al damage would demonstrate elevated levels of alexithymia, relative to other brain-injured patients and healthy control participants. Furthermore, we hypothesized a continuous relationship, whereby the extent of damage to Al would predict alexithymia severity. 


\section{Methods}

\section{Participants}

Data were from Phase 4 of the VHIS (2008-2012), a prospective study of male Vietnam war veterans with penetrating traumatic brain injuries ( $\mathrm{TBI}$ ), and non-brain-injured combat veterans (Raymont et al., 2011). In total, target measures were collected from 129 patients with TBI and 33 control participants. The TBI and control groups were matched with respect to age $\left(M_{\mathrm{ctrl}}=63.33\right.$ years, $\left.S D_{\text {ctrl }}=3.80 ; M_{\mathrm{TBI}}=63.29, S D_{\mathrm{TBI}}=2.89 ; F_{1.160}=0.01, p=0.94, \eta^{2}=0.00 ; B F_{10}=0.21\right)$, education $\left(M_{\mathrm{ctrl}}=15.06\right.$ years, $S D_{\mathrm{ctrl}}=2.12 ; M_{\mathrm{TBI}}=14.55, S D_{\mathrm{TBI}}=2.27 ; F_{1.160}=1.37$, $\left.p=0.24, \eta^{2}=0.01 ; B F_{10}=0.38\right)$, pre-injury intelligence $\left(M_{c t r l}=72.91\right.$ percentile, $\left.S D_{\text {ctrl }}=17.06 ; M_{\mathrm{TBI}}=64.37, S D_{\mathrm{TBI}}=23.29 ; F_{1.136}=2.68, p=0.10, \eta^{2}=0.02 ; B F_{10}=0.76\right)$, and handedness (CTRL group: 26 right-handed, 5 left-handed, 2 ambidextrous; TBI group: 105 right-handed, 22 left-handed, 2 ambidextrous; $X^{2}=2.24, p=0.33$; $\left.B F_{10}=0.06\right)$. An institutional review board at the National Institute of Neurological Disorders and Stroke at the National Institute of Health, Bethesda, MD, USA approved the VHIS Phase 4 protocols, and all participants provided their written informed consent.

\section{Materials}

Lesion localization was performed using the ABLe software package (Solomon et al., 2007) in MEDx v.3.44 (Medical Numerics, Germantown, MD, USA), and an Al atlas was created in MRIcron (Rorden, 2007). For statistical 
analyses, we performed classical hypothesis tests and Bayesian hypothesis tests, as recent advances in quantitative psychology have criticized the practice of wholly relying on $p$-values for statistical evidence (Kass and Raftery, 1995; Kruschke, 2015; Wagenmakers, 2007). All inferential analyses were carried out using the JASP software package (Love et al., 2015). Figure 2 was constructed using the ggplot2 (Wickham, 2009) function implemented in R v.3.2.1 (R Core Team, 2013).

\section{Lesion Localization and Groupings}

Non-contrast axial computerized tomography (CT) scans were acquired during Phase 3 CT scans were acquired during Phase 3 (2003-2006) of the VHIS using a GE Medical Systems Light Speed Plus CT scanner. Six TBI patients in the present sample did not participate in Phase 3, and their CT scans were obtained during Phase 4. On average, patients were scanned 38.18 years after their injury ( $S D=7.96$ years). Magnetic resonance imaging (MRI) could not be performed with patients due to the possible presence of metal fragments from shrapnel or bullet wounds, or residual metallic surgical clips or cranioplasties from surgery. Brain volumes images were composed of $0.4 \mathrm{~mm} \times 0.4 \mathrm{~mm}$ voxels and $2.5 \mathrm{~mm}$ thick overlapping slices with a $1 \mathrm{~mm}$ slice interval. Lesions were manually drawn on CT slices by a trained neuropsychiatrist, and reviewed by a researcher (JG) who was blind to the results of neuropsychological testing. CT images were normalized to a template brain in MNI space. Percent volume loss was calculated by summing the traced lesion areas in each CT slice, multiplying 
by slice thickness and dividing by total brain volume. In this study, we identified percent volume loss to two regions of interest: Al damage (target region) was defined based on a 3D insula template (Afif et al., 2013), and ACC damage (comparison region) was defined using the automated anatomical labeling (AAL) atlas (Tzourio-Mazoyer et al., 2002).

Using the percent volume loss data, we wanted to separate participants into groups based on the extent of their damage to Al. Since small lesion volumes are more amenable to recovery of function (Irle, 1987), it was important to set a relatively high volume threshold, above which it was more likely that any acquired neurocognitive impairments would still be present at testing. Past work has demonstrated that $>15 \%$ damage to a target brain region can be sufficient to induce lasting effects on emotional processes (Koenigs et al., 2008; Tranel et al., 2005). Accordingly, participants were separated into four groups (Figure 1): healthy controls ( $N=33)$, patients with $0 \%$ damage to $\mathrm{Al}(N=89)$, patients with $>0 \%$ and $<15 \%$ damage to $\mathrm{Al}(\mathrm{N}=31)$, and patients with $>15 \%$ damage to $\mathrm{Al}$ $(N=9)$.

\section{Behavioral Measure}

Alexithymia was measured using the twenty item Toronto Alexithymia Scale (TAS-20), the gold standard self-report measure used to index alexithymia (Bagby et al., 1994). The TAS-20 was collected during Phase 4 of the VHIS (2008-2012), an average of 44.34 years ( $S D=7.46$ years) after the injury for the 
patient group. The TAS-20 contains 20 statements with which participants rate their agreement on a 5-point likert scale (1=strongly disagree, 2=disagree, $3=$ =neutral, $4=$ =agree, $5=$ strongly agree). The TAS-20 includes a combination of positively- and negatively-scored items (e.g. positive: "I am often confused about what emotion I am feeling"; e.g. negative: "Being in touch with emotions is essential"), and researchers typically calculate a TAS-20 total score (TAS-total), with higher scores indicating higher levels of alexithymia. Furthermore, the TAS20 can broken down into three subscales: i) difficulty identifying feelings (TASDIF; i.e. related to poor interoceptive and emotional awareness; e.g. "When I am upset, I don't know if I am sad, frightened, or angry," "I am often puzzled by sensations in my body," etc.), ii) difficulty describing feelings (TAS-DDF; i.e. related to poor communication of feelings to others; e.g. "it is difficult for me to find the right words for my feelings," "People often tell me to describe my feelings more," etc.), and iii) externally-oriented thinking (TAS-EXO; i.e. related to a chronic focus on external events over internal states; e.g. "I prefer to analyze problems rather than just describe them," "I prefer talking to people about their daily activities rather than their feelings," etc.; Bagby et al., 1994). Given the hypothesized role of the anterior insula in contributing to emotional awareness, we hypothesized that damage to this region was most likely to influence scores on the TAS-DIF subscale.

Alexithymia covaries with other emotional disturbances, such as anxiety, depression and, of particular relevance to the present sample, post-traumatic 
stress disorder (PTSD; Badura, 2003; Grynberg et al., 2010). Therefore, each of the participants completed measures of trait anxiety (Spielberger et al., 1983), depression (Beck, 1996), and PTSD (Keane et al., 1988), and linear regression models were fitted with TAS-total, TAS-DIF, TAS-DDF, and TAS-EXO as dependent variables, and the three control measures as predictors. The standardized residuals from these four models indexed the various dimensions of alexithymia after controlling for individual differences in anxiety, depression, and PTSD. These standardized alexithymia measures were used in all inferential analyses.

\section{Results}

\section{Analyses of Variance}

We began by performing an ANOVA to determine whether pronounced damage to Al causes elevated levels of alexithymia, as indexed using the TAStotal score. The overall ANOVA was significant $\left(F_{3,158}=3.98, p=0.009, \eta^{2}=0.07\right.$; $B F_{10}=2.20$ ), providing evidence that there were group differences in alexithymia. Planned independent samples $t$-tests and Bayesian $t$-tests suggested that the group with pronounced (i.e. >15\%) Al damage was associated with a significant increase in alexithymia relative to healthy control participants $\left(t_{40}=2.84, p=0.007\right.$, $d=1.07, B F_{10}=6.38$; Figure 2a), patients with no Al damage $\left(t_{96}=3.43, p<0.001\right.$, $d=1.20, B F_{10}=31.61 ;$ Figure $\left.2 a\right)$, and patients with some Al damage $\left(t_{38}=2.60\right.$, $p=0.01, d=0.98, B F_{10}=4.02 ;$ Figure 2a). 
Next, we performed ANOVAs on the three TAS-20 subscales to determine whether Al damage was specifically associated with heightened scores on the "difficulty identifying feelings" subscale (TAS-DIF). The TAS-DIF ANOVA provided evidence for significant differences between groups $\left(F_{3,158}=4.91\right.$, $p=0.003, \eta^{2}=0.08 ; B F_{10}=5.13$; Figure $2 b$ ). Specifically, TAS-DIF levels were significantly higher in patients with large Al lesions relative to healthy controls $\left(t_{40}=4.40, p<0.001, d=1.65, B F_{10}=240.36\right.$; Figure $\left.2 b\right)$, patients with no damage to Al $\left(t_{96}=3.29, p=0.001, d=1.15, B F_{10}=21.66\right.$; Figure $\left.2 b\right)$, and patients with minimal damage to $\mathrm{Al}\left(t_{38}=2.84, p=0.007, d=1.08, B F_{10}=6.38\right.$; Figure $\left.2 \mathbf{b}\right)$. In contrast, results for the "difficulty describing feelings" (TAS-DDF) and "externally-oriented thinking" (TAS-EXO) provided no evidence for a relationship with Al damage. Neither the TAS-DDF ANOVA $\left(F_{3,158}=2.35, p=0.07, \eta^{2}=0.04 ; B F_{10}=0.48\right.$; Figure 2c), nor the TAS-EXO ANOVA $\left(F_{3,158}=1.58, p=0.20, \eta^{2}=0.03 ; B F_{10}=0.27\right.$; Figure 2d), provided credible evidence for a difference between groups. Therefore, in accordance with our predictions, pronounced Al damage caused increased levels of alexithymia, and this effect was primarily driven by differences in the TAS-DIF subscale.

\section{Regression Analyses}

Next, we conducted classical and Bayesian linear regression analyses to provide further evidence that damage to Al was specifically associated with alexithymia. Both $\mathrm{Al}$ and $\mathrm{ACC}$ damage were included in the model. Al and ACC 
are among the most commonly co-activated structures in the functional MRI (fMRI) literature on emotional processing (Craig, 2009; Medford and Critchley, 2010), and are often both damaged in lesion patients (Damasio et al., 2013; Khalsa et al., 2009), making it difficult to discern the differential contribution of each region. The initial regression model included the standardized TAS-total score as the dependent variable, and included percent damage to Al, percent damage to ACC, and total percent damage as regressors (Table 2), which were all $T$-scored in an attempt to reduce multicollinearity. Overall, the model explained a significant portion of variance in alexithymia $\left(R^{2}=0.10, F_{3,125}=4.39\right.$, $p=0.006, f^{2}=0.10, B F_{10}=4.32$; Table 2), with $\mathrm{Al}$ damage predicting a significant increase in alexithymia $\left(\beta=0.02 ; t_{125}=2.38, p=0.01\right.$, one-tailed, $B F_{10}=8.82$; Table 2). In contrast, neither $A C C$ damage ( $p=0.07$, one-tailed, $B F_{10}=0.71$; Table 2$)$ nor total lesion volume ( $p=0.33$, one-tailed, $B F_{10}=0.31$; Table 2$)$ contributed significantly to the model. Importantly, none of the regressors demonstrated an issue with multicollinearity (all VIF $<2$ ).

Akin to our ANOVA procedures, we also conducted separate linear regression analyses for each of the TAS-20 subscales. A significant amount of variance on the TAS-DIF subscale was accounted for by our regression model $\left(R^{2}=0.09, F_{3,125}=4.25, p=0.007, f^{2}=0.10, B F_{10}=3.67 ;\right.$ Table 2$)$. Increases in $\mathrm{Al}$ damage predicted increases in TAS-DIF $\left(\beta=0.02 ; t_{125}=1.86, p=0.03\right.$, one-tailed, $B F_{10}=4.04$; Table 2), whereas the $A C C$ damage $\left(p=0.05\right.$, one-tailed, $B F_{10}=1.00$;

Table 2) and total volume loss variables ( $p=0.44$, one-tailed, $B F_{10}=0.39$; Table 2) 
did not contribute significantly to the model. In contrast, the models did not account for a significant proportion of variance in the TAS-DDF $\left(R^{2}=0.04\right.$, $F_{3,125}=1.86, p=0.14, f^{2}=0.04, B F_{10}=0.19$; Table 2$)$ and TAS-EXO $\left(R^{2}=0.04\right.$, $F_{3,125}=1.76, p=0.16, f^{2}=0.04, B F_{10}=0.17$; Table 2) subscales.

\section{Control Analyses}

To ensure that our key ANOVA results were specific to alexithymia and not some unanticipated factor, we compared the groups on a variety of demographic and control measures (Table 1). The only measures that showed slight differences between groups were pre- and post-injury intelligence, and we entered these variables into an analysis of covariance similar to our original ANOVA. Again, there was a significant difference between the groups $\left(F_{3,156}=3.08, p=0.03, \eta^{2}=0.06\right)$, suggesting that group differences in intelligence could not account for our findings.

Lastly, since the TAS-20 asks participants to perform a metacognitive appraisal (e.g. "I often don't know why I am angry"), this might be problematic if they are not aware of their own interoceptive or emotional difficulties (Lane et al., 2015). Therefore, we wanted to ensure that TAS-20 performance was negatively associated with a measure of emotional awareness that did not rely on metacognition. Accordingly, a subsample of our participants $(N=135)$ completed the Mayer-Salovey-Caruso Emotional Intelligence Scale (MSCEIT; Mayer et al., 2002), a self-report measure that asks participants to interpret emotional stimuli 
rather than performing an appraisal of their own emotions. Crucially, the "perceiving emotions" and "understanding emotions" subscales of the MSCEIT were negatively associated with raw TAS-total scores (perceiving emotions: $r=-$ $0.18, p=0.02, B F_{10}=1.05$; understanding emotions: $r=-0.24, p=0.003, B F_{10}=4.66$ ), supporting the construct validity of our alexithymia scale.

\section{Discussion}

In the present study, we examined whether damage to Al can cause acquired alexithymia. Recent computational models suggest that interoceptive signals are integrated by $\mathrm{Al}$ to produce a cohesive emotional awareness state (Gu et al., 2013; Seth, 2013) and, by extension, that impaired Al processing might be involved in producing alexithymia (Brewer et al., 2015b; Quattrocki and Friston, 2014). The present data represent compelling evidence in support of this hypothesis, demonstrating that pronounced damage to Al causes impaired awareness of emotional states in the self. The present study also helps to distinguish between $\mathrm{Al}$ and $\mathrm{ACC}$ involvement in emotional cognition. $\mathrm{Al}$ and $\mathrm{ACC}$ are commonly co-activated in the fMRI literature (Craig, 2009; Medford and Critchley, 2010), and are both compromised in most Al lesion studies (Damasio et al., 2013; Khalsa et al., 2009), making it difficult to demonstrate clear dissociations between their functions. Here, using a multiple linear regression approach, we present preliminary evidence for a rare dissociation between the 
two regions: Al damage is associated with elevated levels of alexithymia whereas ACC damage is not.

Alexithymia has traditionally been studied with respect to its comorbidity with neurodevelopmental disorders such as autism spectrum disorders (Bird and Cook, 2013) and schizophrenia (Fogley et al., 2014), but recent studies have suggested that alexithymia can be acquired following brain injury (Henry et al., 2006; Williams and Wood, 2013, 2010). The present findings extend this work by showing that alexithymia can be acquired following injuries to the Al specifically, with more pronounced lesions being associated with greater impairment. This finding provides causal support for the view that abnormal Al recruitment may be a key neurophysiological feature of developmental alexithymia (Brewer et al., 2015b; Quattrocki and Friston, 2014).

The present evidence for acquired alexithymia following Al damage is in agreement with a considerable neuroimaging literature (Craig, 2009), and several human lesion studies implicating $\mathrm{Al}$ in interoception and emotional awareness (Gu et al., 2013; Jones et al., 2010). For example, Al grey matter volumes (Borsci et al., 2009; Ihme et al., 2013) and functional activations are reduced in alexithymic individuals (Bird et al., 2010; Kano et al., 2003; Reker et al., 2010; Silani et al., 2008). Within the broader alexithymic construct, the present findings suggest that $A l$ is specifically implicated in emotional awareness (i.e. TAS-DIF subscale). This conclusion is in line with other human lesion studies, which have 
found $\mathrm{Al}$ damage to be associated with reduced sensitivity to aversive somatosensory stimuli (Greenspan et al., 1999), and a muted experience of disgust (Calder et al., 2000). These converging lines of evidence support the arguments that Al produces an emotional awareness state by integrating ascending interoceptive signals (Gu et al., 2013; Seth, 2013), and that this system is disrupted in alexithymia (cf. Brewer et al., 2015b; Herbert et al., 2011; Quattrocki and Friston, 2014).

In contrast to the present findings, recent case studies have documented relatively intact performance on tasks related to conscious awareness, emotion, and interoception in patients with Al lesions (Damasio et al., 2013; Feinstein et al., in press; Khalsa et al., 2009; Philippi et al., 2012). However, these studies are based on two patients (Patient $B$ and Patient R), raising the possibility that these individuals have developed compensatory strategies for coping with impaired emotional awareness that were not deployed by the present sample of $\mathrm{Al}$ patients. Additionally, none of the previous Al lesion studies measured alexithymia using the TAS-20, and it is possible that the TAS-20 measures dimensions of emotional awareness that require a functioning $\mathrm{Al}$, whereas the other behavioral tasks used in previous studies did not. By showing significant increases in alexithymia in a group of patients with Al damage $(N=9)$, and a specific association between the extent of Al damage and increased alexithymia, our data provide evidence that $\mathrm{Al}$ is indeed necessary for the dimensions of emotional awareness that are disrupted in alexithymia. 
The present findings also appear to contradict a Positron Emission Tomography study by Lane and colleagues (1998), which found that ACC activity during emotional experience was positively associated with scores on the "Levels of Emotional Awareness Scale" (LEAS; Lane et al., 1990) in healthy volunteers (Lane et al., 1998). In contrast, the present findings suggest that emotional awareness is not disrupted in brain-injured patients as a function of ACC damage, beyond the variance accounted for by Al damage. The reason(s) for this discrepancy are unclear, but it seems likely that the LEAS and TAS-20 reflect different aspects of emotional awareness that differentially recruit the ACC and $\mathrm{Al}$, respectively. Future functional neuroimaging studies will be required to further dissociate the emotional awareness operations of Al and ACC.

One potential limitation of the present study was that we relied on an alexithymia measure (the TAS-20) that required participants to have insight into their own emotional awareness difficulties. Fortunately, the negative association between our alexithymia data and the more objective MSCEIT supports the construct validity of the TAS-20. In future Al lesion-symptom mapping studies, researchers should aim to provide conceptual replications of the present findings using more objective measures of emotional awareness. An additional caveat to our findings is that our sample was limited to older male combat veterans studied approximately 45 years post-injury. 
We believe that our findings, notwithstanding the caveats articulated above, provide crucial evidence for the importance of $\mathrm{Al}$ in emotional awareness. Future studies should work to clarify how emotional states are linked with the ongoing cognitive and social context within conscious awareness - an important step in determining the personal relevance of daily experiences. 


\section{References}

Afif, A., Becq, G., Mertens, P., 2013. Definition of a stereotactic 3-dimensional magnetic resonance imaging template of the human insula. Neurosurgery 72, ons35-ons46. doi:10.1227/NEU.0b013e31826cdc57

Aleman, A., 2005. Feelings you can't imagine: Towards a cognitive neuroscience of alexithymia. Trends Cogn. Sci. 9, 553-555. doi:10.1016/j.tics.2005.10.003

Badura, A.S., 2003. Theoretical and empirical exploration of the similarities between emotional numbing in posttraumatic stress disorder and alexithymia. J. Anxiety Disord. 17, 349-360. doi:10.1016/S08876185(02)00201-3

Bagby, R.M., Parker, J.D., Taylor, G.J., 1994. The twenty-item Toronto alexithymia scale--I. Item selection and cross-validation of the factor structure. J. Psychosom. Res. 38, 23-32. doi:10.1016/0022-3999(94)900051

Beck, A.T., 1996. Manual for the Beck Depression Inventory-II. The Psychological Corporation, San Antonio, TX.

Bernhardt, B.C., Valk, S.L., Silani, G., Bird, G., Frith, U., Singer, T., 2014. Selective Disruption of Sociocognitive Structural Brain Networks in Autism and Alexithymia. Cereb. Cortex 24, 3258-3267. doi:10.1093/cercor/bht182

Bird, G., Cook, R., 2013. Mixed emotions: The contribution of alexithymia to the emotional symptoms of autism. Transl. Psychiatry 3, e285. doi:10.1038/tp.2013.61

Bird, G., Silani, G., Brindley, R., White, S., Frith, U., Singer, T., 2010. Empathic 
brain responses in insula are modulated by levels of alexithymia but not autism. Brain 133, 1515-1525. doi:10.1093/brain/awq060

Borsci, G., Boccardi, M., Rossi, R., Rossi, G., Perez, J., Bonetti, M., Frisoni, G.B., 2009. Alexithymia in healthy women: A brain morphology study. J. Affect. Disord. 114, 208-215. doi:10.1016/j.jad.2008.07.013

Brewer, R., Cook, R., Cardi, V., Treasure, J., Bird, G., 2015a. Emotion recognition deficits in eating disorders are explained by co-occurring alexithymia. R. Soc. Open Sci. 2, 140382.

Brewer, R., Happé, F., Cook, R., Bird, G., 2015b. Commentary on "Autism, oxytocin and interoception.": Alexithymia, not autism spectrum disorders, is the consequence of interoceptive failure. Neurosci. Biobehav. Rev. 56, 348353.

Calder, A.J., Keane, J., Manes, F., Antoun, N., Young, A.W., 2000. Impaired recognition and experience of disgust following brain injury. Nat. Neurosci. 3, 1077-1078. doi:10.1038/80586

Craig, A.D., 2009. How do you feel--now? The anterior insula and human awareness. Nat. Rev. Neurosci. 10, 59-70. doi:10.1038/nrn2555

Damasio, A., Damasio, H., Tranel, D., 2013. Persistence of feelings and sentience after bilateral damage of the insula. Cereb. Cortex 23, 833-846. doi:10.1093/cercor/bhs077

Feinstein, J.S., Khalsa, S.S., Salomons, T. V., Prkachin, K.M., Frey-Law, L.A., Lee, J.E., Tranel, D., Rudrauf, D., n.d. Preserved emotional awareness of pain in a patient with extensive bilateral damage to the insula, anterior 
cingulate, and amygdala. Brain Struct. Funct. doi:10.1007/s00429-014-09863

FeldmanHall, O., Dalgleish, T., Mobbs, D., 2013. Alexithymia decreases altruism in real social decisions. Cortex 49, 899-904.

doi:10.1016/j.cortex.2012.10.015

Fogley, R., Warman, D., Lysaker, P.H., 2014. Alexithymia in schizophrenia:

Associations with neurocognition and emotional distress. Psychiatry Res. 218, 1-6. doi:10.1016/j.psychres.2014.04.020

Grafman, J., Jonas, B., Martin, A., Salazar, A. M., Weingartner, H., Ludlow, C., Smutok, M.A., Vance, S.C., 1988. Intellectual function following penetrating head injury in Vietnam veterans. Brain 111, 169-184.

doi:http://dx.doi.org/10.1093/brain/111.1.169

Greenspan, J.D., Lee, R.R., Lenz, F.A., 1999. Pain sensitivity alterations as a function of lesion location in the parasylvian cortex. Pain 81, 273-282. doi:10.1016/S0304-3959(99)00021-4

Grynberg, D., Luminet, O., Corneille, O., Grèzes, J., Berthoz, S., 2010.

Alexithymia in the interpersonal domain: A general deficit of empathy? Pers. Individ. Dif. 49, 845-850. doi:10.1016/j.paid.2010.07.013

Gu, X., Hof, P.R., Friston, K.J., Fan, J., 2013. Anterior insular cortex and emotional awareness. J. Comp. Neurol. 521, 3371-3388. doi:10.1002/cne.23368

Henry, J.D., Phillips, L.H., Crawford, J.R., Theodorou, G., Summers, F., 2006. Cognitive and psychosocial correlates of alexithymia following traumatic 
brain injury ๕. Neuropsychologia 44, 62-72.

doi:10.1016/j.neuropsychologia.2005.04.011

Herbert, B.M., Herbert, C., Pollatos, O., 2011. On the relationship between interoceptive awareness and alexithymia: Is interoceptive awareness related to emotional awareness? J. Pers. 79, 1149-1175. doi:10.1111/j.14676494.2011.00717.x

Ihme, K., Dannlowski, U., Lichev, V., Stuhrmann, A., Grotegerd, D., Rosenberg, N., Kugel, H., Heindel, W., Arolt, V., Kersting, A., Suslow, T., 2013. Alexithymia is related to differences in gray matter volume: A voxel-based morphometry study. Brain Res. 1491, 60-67. doi:10.1016/j.brainres.2012.10.044

Irle, E., 1987. Lesion size and recovery of function: Some new perspectives. Brain Res. Rev. 12, 307-320. doi:10.1016/0165-0173(87)90003-8 Jones, C.L., Ward, J., Critchley, H.D., 2010. The neuropsychological impact of insular cortex lesions. J. Neurol. Neurosurg. Psychiatry 81, 611-618. doi:10.1136/jnnp.2009.193672

Kano, M., Fukudo, S., Gyoba, J., Kamachi, M., Tagawa, M., Mochizuki, H., Itoh, M., Hongo, M., Yanai, K., 2003. Specific brain processing of facial expressions in people with alexithymia: An H215O-PET study. Brain 126, 1474-1484. doi:10.1093/brain/awg131

Kass, R.E., Raftery, A.E., 1995. Bayes Factors. J. Am. Stat. Assoc. 90, 773-795. doi:doi: $10.2307 / 2291091$

Keane, T.M., Caddell, J.M., Taylor, K.L., 1988. Mississippi scale for combat- 
related posttraumatic stress disorder: Three studies in reliability and validity.

J. Consult. Clin. Psychol. 56, 85-90. doi:10.1037//0022-006X.56.1.85

Khalsa, S.S., Rudrauf, D., Feinstein, J.S., Tranel, D., 2009. The pathways of interoceptive awareness. Nat. Neurosci. 12, 1494-1496.

doi:10.1016/j.biotechadv.2011.08.021.Secreted

Koenigs, M., Huey, E.D., Raymont, V., Cheon, B., Solomon, J., Wasserman, E.M., Grafman, J., 2008. Focal brain damage protects against post-traumatic stress disorder in combat veterans. Nat. Neurosci. 11, 232-237. doi:10.1038/nn2032

Kruschke, J.K., 2015. Doing bayesian data analysis: A tutorial with R, JAGS, and Stan, 2nd ed. Academic Press, San Diego, CA.

Lane, R.D., Quinlan, D.M., Schwartz, G.E., Walker, P.A., Zeitlin, S.B., 1990. The Levels of Emotional Awareness Scale: a cognitive-developmental measure of emotion. J. Pers. Assess. 55, 124-134.

doi:10.1207/s15327752jpa5501\&2_12

Lane, R.D., Reiman, E.M., Axelrod, B., Yun, L.S., Holmes, A., Schwartz, G.E., 1998. Neural correlates of levels of emotional awareness: Evidence of an interaction between emotion and attention in the anterior cingulate cortex. J. Cogn. Neurosci. 10, 525-535. doi:10.1162/089892998562924

Lane, R.D., Weihs, K.L., Herring, A., Hishaw, A., Smith, R., 2015. Affective agnosia: Expansion of the alexithymia construct and a new opportunity to integrate and extend Freud's legacy. Neurosci. Biobehav. Rev. 55, 594-611. doi:10.1016/j.neubiorev.2015.06.007 
Love, J., Selker, R., Marsman, M., Jamil, T., Verhagen, A.J., Ly, A., Gronau, Q.F., Smira, M., Epskamp, S., Matzke, D., Wild, A., Rouder, J.N., Morey, R.D., Wagenmakers, E.J., 2015. JASP.

Mayer, J.D., Salovey, P., Caruso, D.R., Sitarenios, G., 2002. Mayer-SaloveyCaruso Emotional Intelligence Test (Version 2.0) User's Manual. MultiHealth Systems, Toronto, Canada.

Medford, N., Critchley, H.D., 2010. Conjoint activity of anterior insular and anterior cingulate cortex: awareness and response. Brain Struct. Funct. 115. doi:10.1007/s00429-010-0265-x

Nemiah, J.C., Freyberger, H., Sifneos, P.E., 1976. Alexithymia: A view of the psychosomatic process. Mod. Trends Psychosom. Med. 3, 430-439.

Philippi, C.L., Feinstein, J.S., Khalsa, S.S., Damasio, A., Tranel, D., Landini, G., Williford, K., Rudrauf, D., 2012. Preserved self-awareness following extensive bilateral brain damage to the insula, anterior cingulate, and medial prefrontal cortices. PLoS One 7, e38413. doi:10.1371/journal.pone.0038413 Quattrocki, E., Friston, K., 2014. Autism, oxytocin and interoception. Neurosci. Biobehav. Rev. 47, 410-430. doi:10.1016/j.neubiorev.2014.09.012

Raymont, V., Salazar, A.M., Krueger, F., Grafman, J., 2011. "Studying injured minds" - the Vietnam head injury study and 40 years of brain injury research. Front. Neurol. 2, 15. doi:10.3389/fneur.2011.00015

Reker, M., Ohrmann, P., Rauch, A. V., Kugel, H., Bauer, J., Dannlowski, U., Arolt, V., Heindel, W., Suslow, T., 2010. Individual differences in alexithymia and brain response to masked emotion faces. Cortex 46, 658-667. 
doi:10.1016/j.cortex.2009.05.008

Robson, D., 2015. What is it like to have never felt an emotion? BBC News.

Rorden, C., 2007. MRIcron.

Serani, D., 2014. The emotional blindness of alexithymia. Sci. Am.

Seth, A.K., 2013. Interoceptive inference, emotion, and the embodied self.

Trends Cogn. Sci. 17, 565-573. doi:10.1016/j.tics.2013.09.007

Silani, G., Bird, G., Brindley, R., Singer, T., Frith, C., Frith, U., 2008. Levels of emotional awareness and autism: An fMRI study. Soc. Neurosci. 3, 97-112. doi:10.1080/17470910701577020

Solomon, J., Raymont, V., Braun, A., Butman, J.A., Grafman, J., 2007. Userfriendly software for the analysis of brain lesions (ABLe). Comput. Methods Programs Biomed. 86, 245-254. doi:10.1016/j.cmpb.2007.02.006

Spielberger, C.D., Gorsuch, R.L., Lushene, R., Vagg, P.R., Jacobs, G.A., 1983. Manual for the state-trait anxiety inventory. Consulting Psychologists Press, Palo Alto, CA.

Taylor, G.J., Bagby, R.M., Parker, J.D.A., 1991. The alexithymia construct: A potential paradigm for psychosomatic medicine. Psychosomatics 32, 153164.

Team, R.C., 2013. R: A Language and Environment for Statistical Computing.

Tranel, D., Damasio, H., Denburg, N.L., Bechara, A., 2005. Does gender play a role in functional asymmetry of ventromedial prefrontal cortex? Brain 128, 2872-2881. doi:10.1093/brain/awh643

Tzourio-Mazoyer, N., Landeau, B., Papathanassiou, D., Crivello, F., Etard, O., 
Delcroix, N., Mazoyer, B., Joliot, M., 2002. Automated anatomical labeling of activations in SPM using a macroscopic anatomical parcellation of the MNI MRI single-subject brain. Neuroimage 15, 273-289.

doi:10.1006/nimg.2001.0978

Wagenmakers, E.J., 2007. A practical solution to the pervasive problems of $p$ values. Psychon. Bull. Rev. 14, 779-804.

Wechsler, D., 1997. Wechsler Memory Scale, 3rd ed. The Psychological Corporation, San Antonio, TX.

Wickham, H., 2009. ggplot2: Elegant graphics for data analysis. Springer, New York.

Williams, C., Wood, R.L., 2013. The impact of alexithymia on relationship quality and satisfaction following traumatic brain injury. J. Head Trauma Rehabil. 28, 16-19. doi:10.1097/HTR.0b013e318267b0ab

Williams, C., Wood, R.L., 2010. Alexithymia and emotional empathy following traumatic brain injury. J. Clin. Exp. Neuropsychol. 32, 259-267. doi:10.1080/13803390902976940 


\section{Tables}

Table 1. Demographics from the three groups in the initial ANOVA analyses. ${ }^{* * *}$ : Significant at the $p<0.001$ level; $^{T}$ : Trending at $p<0.1$. Age and education are listed in the number of years. Pre-injury intelligence is a percentile score on the Armed-Forces Qualification Test (AFQT-7A), which was collected at induction to the military and is highly correlated with the Wechsler Adult Intelligence Scale-III (WAIS-III; Grafman et al., 1988). Post-injury intelligence was measured using percentile scores on the WAIS-III (Wechsler, 1997) at the time of evaluation. Missing values for both the pre-injury intelligence and post-injury intelligence scores were filled using a mean replacement method. Lastly, given their potential association with alexithymia, we also analyzed raw scores from the Beck Depression Inventory-II (Beck, 1996), the State-Trait Anxiety Inventory (Spielberger et al., 1983), and the Mississippi PTSD scale (Keane et al., 1988).

\begin{tabular}{|c|c|c|c|c|c|c|c|c|c|}
\hline & \multicolumn{2}{|c|}{ Controls } & \multicolumn{2}{|c|}{$0 \%$ AI } & \multicolumn{2}{|c|}{$<15 \%$ AI } & \multicolumn{2}{|c|}{$>15 \% \mathrm{AI}$} & Inferential Tests \\
\hline \multicolumn{10}{|c|}{ Demographics: } \\
\hline Age & 63.33 & $(3.80)$ & 63.38 & $(3.15)$ & 62.97 & $(2.17)$ & 63.44 & $(2.45)$ & $\begin{array}{c}F_{3.158}=0.15, p=0.93 \\
\eta^{2}=0.003 ; B F_{10}=0.06\end{array}$ \\
\hline Education & 15.06 & $(2.12)$ & 14.60 & $(2.29)$ & 14.68 & $(2.01)$ & 13.67 & $(2.92)$ & $\begin{array}{c}F_{3.158}=0.96, p=0.41, \eta^{2}=0.02 ; \\
B F_{10}=0.12\end{array}$ \\
\hline $\begin{array}{l}\text { Handedness } \\
(R, L, A)\end{array}$ & \multicolumn{2}{|c|}{$(26,5,2)$} & \multicolumn{2}{|c|}{$(69,18,2)$} & \multicolumn{2}{|c|}{$(28,3,0)$} & \multicolumn{2}{|c|}{$(8,1,0)$} & $\begin{array}{c}X^{2}=5.08, p=0.53 \\
B F_{10}=2.65 \mathrm{e}-4\end{array}$ \\
\hline \multicolumn{10}{|c|}{ Control Measures: } \\
\hline $\begin{array}{c}\text { Pre-injury } \\
\text { Intelligence }^{T}\end{array}$ & 70.52 & $(14.24)$ & 63.28 & $(23.20)$ & 70.61 & (16.66) & 55.67 & $(24.37)$ & $\begin{array}{c}F_{3.158}=2.32, p=0.08, \eta^{2}=0.04 \\
B F_{10}=0.56\end{array}$ \\
\hline $\begin{array}{c}\text { Post-injury } \\
\text { Intelligence }\end{array}$ & 75.28 & $(21.45)$ & 66.68 & $(26.37)$ & 61.39 & $(25.11)$ & 28.73 & $(31.31)$ & $\begin{array}{c}F_{3.158}=8.19, p<0.001 \\
\eta^{2}=0.14 ; B F_{10}=185.55\end{array}$ \\
\hline Depression & 10.64 & $(8.74)$ & 7.96 & $(7.67)$ & 7.55 & $(9.44)$ & 6.11 & (6.68) & $\begin{array}{c}F_{3.158}=1.24, p=0.30, \eta^{2}=0.02 \\
B F_{10}=0.18\end{array}$ \\
\hline Anxiety & 37.55 & $(12.45)$ & 33.65 & $(10.14)$ & 33.26 & (11.54) & 31.11 & $(7.37)$ & $\begin{array}{c}F_{3.158}=1.46, p=0.23, \eta^{2}=0.03 ; \\
B F_{10}=0.23\end{array}$ \\
\hline PTSD & 83.67 & $(22.88)$ & 79.79 & $(22.70)$ & 76.26 & (18.68) & 74.33 & (19.58) & $\begin{array}{c}F_{3.158}=0.80, p=0.50, \eta^{2}=0.02 \\
B F_{10}=0.11\end{array}$ \\
\hline
\end{tabular}


Table 2. Results from the regression analysis. *: Regressor is significant at the $p<0.05$ (one-tailed) and $B F_{10}>3$ level.

\begin{tabular}{|c|c|c|c|}
\hline Regressor & $\beta$ & t-Test & $B F_{10}$ \\
\hline \multicolumn{4}{|c|}{ TAS-DIF ("Difficulty Identifying Feelings") } \\
\hline$T-\mathrm{Al}^{*}$ & 0.02 & $t=1.86, p=0.03$ & 4.04 \\
\hline T-ACC & 0.01 & $t=1.64, p=0.05$ & 1.00 \\
\hline$T$-Volume Loss & 0.005 & $T=0.14, p=0.44$ & 0.39 \\
\hline \multicolumn{4}{|c|}{ Overall Model ${ }^{* *}: R^{2}=0.09 ; F_{3,125}=4.25, p=0.007, f^{2}=0.10 ; B F_{10}=3.67$} \\
\hline \multicolumn{4}{|c|}{ TAS-DDF ("Difficulty Describing Feelings") } \\
\hline T-AI & 0.02 & $t=1.78, p=0.04$ & 1.79 \\
\hline T-ACC & 0.004 & $t=0.50, p=0.31$ & 0.31 \\
\hline$T$-Volume Loss & -0.01 & $t=-0.31, p=0.38$ & 0.33 \\
\hline \multicolumn{4}{|c|}{ Overall Model: $R^{2}=0.04 ; F_{3,125}=1.86, p=0.14, f^{2}=0.04 ; B F_{10}=0.19$} \\
\hline \multicolumn{4}{|c|}{ TAS-EXO (“Externally-Oriented Thinking”) } \\
\hline T-AI & 0.02 & $t=1.70, p=0.04$ & 0.94 \\
\hline T-ACC & 0.009 & $t=1.11, p=0.13$ & 0.50 \\
\hline$T$-Volume Loss & -0.03 & $t=-0.84, p=0.20$ & 0.30 \\
\hline \multicolumn{4}{|c|}{ Overall Model: $R^{2}=0.04 ; F_{3,125}=1.76, p=0.16, f^{2}=0.04 ; B F_{10}=0.17$} \\
\hline \multicolumn{4}{|c|}{ TAS-total } \\
\hline$T-A I^{*}$ & 0.02 & $t=2.38, p=0.009$ & 8.82 \\
\hline T-ACC & 0.01 & $t=1.50, p=0.07$ & 0.71 \\
\hline$T$-Volume Loss & -0.01 & $t=-0.44, p=0.33$ & 0.31 \\
\hline
\end{tabular}




\section{Figures}

Figure 1. Representative axial slices depicting (A) the template used to define the insula (pink circles approximately marking the anterior sector), and (B) overlay density maps depicting the preserved anterior insula (Al) in the no damage group ( $N=89)$, some slight damage to $\mathrm{Al}$ in the $<15 \%$ group $(N=31)$, and pronounced damage in the $>15 \%$ group $(N=9)$. Images are in radiological space (i.e. right is left).

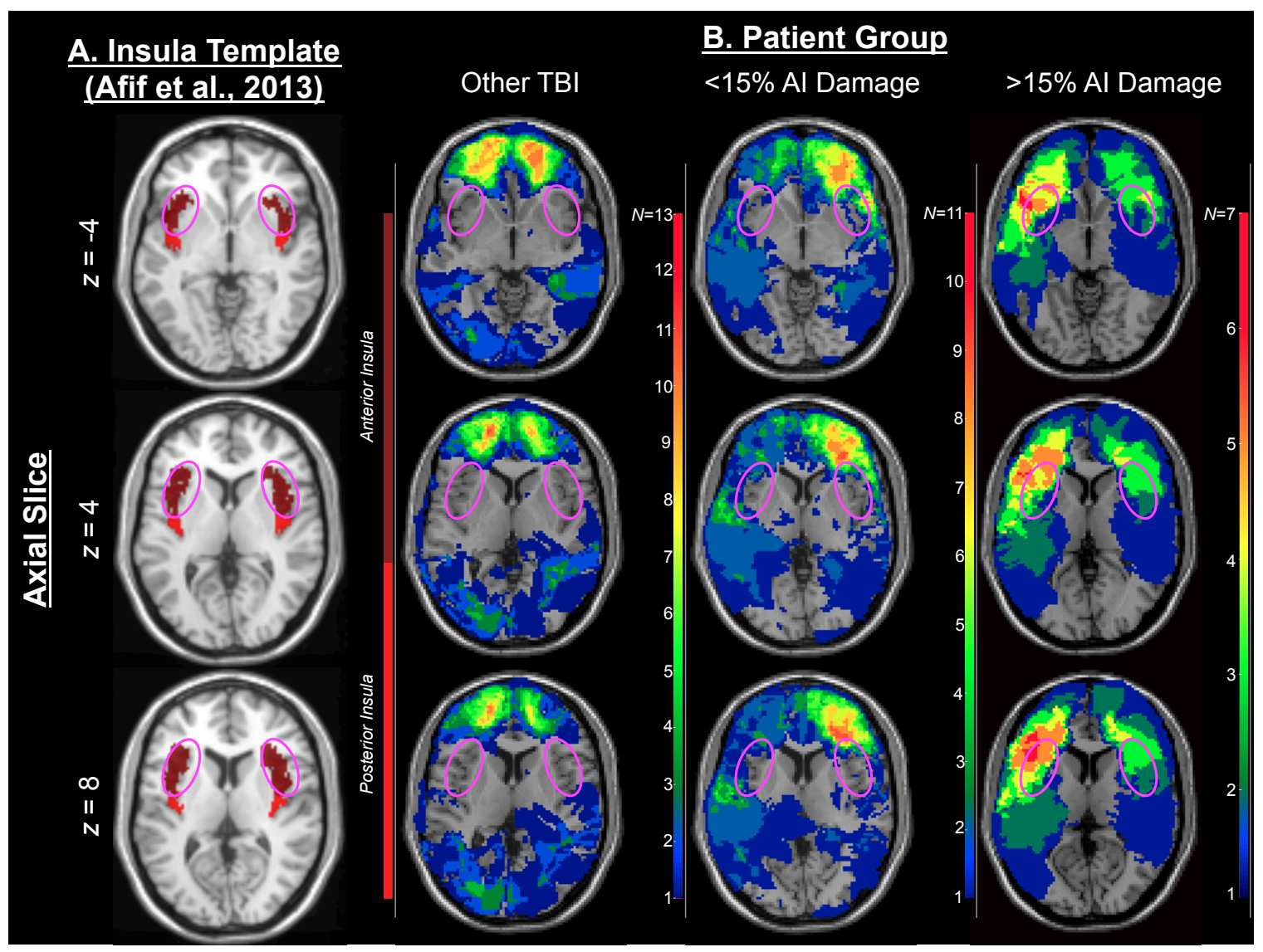


Figure 2. (A) Patients with pronounced damage to Al demonstrated significant increases in alexithymia (TAS-total) relative to all other patient groups as well as healthy controls. (B) Scores on the "difficulty identifying feelings" (DIF) subscale mirrored the TAS-total results, whereas (C-D) neither the "difficulty describing feelings (DDF) nor the "externally-oriented thinking" (EXO) subscales varied significantly as a function of group. ${ }^{* * *}: p<0.001,{ }^{* *}: p<0.01 ; n s$ : ANOVA $p>0.05$.
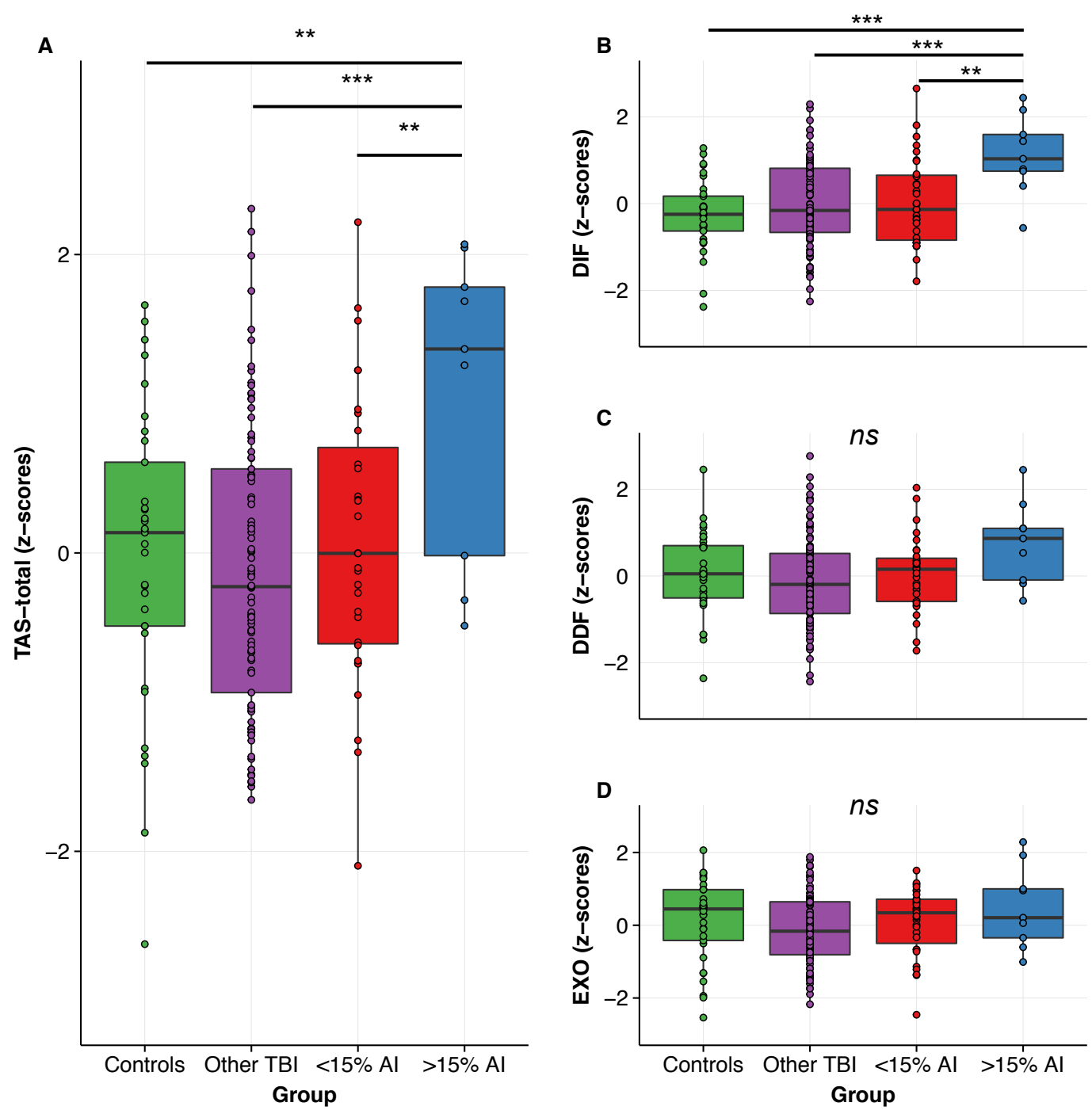